\title{
CurD: A Tool for Diffusion Analyses on Curved Membranes
}

\author{
Balázs Fábián ${ }^{*, \dagger, \ddagger}$ and Matti Javanainen ${ }^{\dagger, \uparrow}$ \\ $\dagger$ Institute of Organic Chemistry and Biochemistry of the Czech Academy of Sciences, \\ Flemingovo nám. 542/2, CZ-16000 Prague 6, Czech Republic \\ $\ddagger$ Department of Theoretical Biophysics, Max Planck Institute of Biophysics, Max-von-Laue \\ Straße 3, 60438, Frankfurt am Main, Germany \\ \Institute of Biotechnology, University of Helsinki, FI-00014 University of Helsinki, \\ Finland
}

E-mail: balazs.fabian@biophys.mpg.de 


\begin{abstract}
Curved membranes are abundant and functionally relevant in living matter, yet they have eluded computational studies due to methodological limitations. With multiple approaches available for setting up simulations on such curved membranes, there is a growing need for efficient and versatile tools to analyze their outcomes. Here, we present CurD, a freely available tool for analyzing the diffusion of membrane constituents along curved surfaces. The tool efficiently uses the Vertex-oriented Triangle Propagation algorithm to compute geodesic distances significantly faster than conventional implementations of path-finding algorithms, while providing a friendly command-line interface. With CurD, we resolve the effects of curvature and lipid packing densities on the diffusion of lipids on two curved membranes - one with only mean curvature and another with both Mean and Gaussian curvature. We find that Gaussian curvature plays a surprisingly small role, whereas mean curvature or packing of lipid headgroups largely dictates their mobility.
\end{abstract}

\title{
Graphical TOC Entry
}

\begin{tabular}{|l|}
\hline TOC ENTRY REQUIRED \\
\hline
\end{tabular}


Molecular simulations are starting to achieve sufficient length- and time-scales that allow us to efficiently probe the compositional and topological complexity of real cellular membranes. ${ }^{1-3}$ Notably, many key functions related to these membranes encapsulating either the entire cell or its organelles involve significant local membrane curvature. ${ }^{4}$ The plasma membrane has specific invaginated signaling platforms, ${ }^{5}$ the complex and dynamic topography of the mitochondrial inner membrane is involved in numerous processes, ${ }^{6}$ and the cells store energy in lipid droplets that bud from endoplasmic reticulum. ${ }^{7}$ In these processes, curvature is generated by both lipids ${ }^{8}$ and proteins, ${ }^{9}$ yet they both also sense curvature, leading to their spatial sorting with functional implications. ${ }^{10,11}$

Adequate modelling ${ }^{12}$ and analysis ${ }^{13}$ of such non-planar lipid bilayers requires the development of algorithms that take into account the geometry of the membrane. A particularly fascinating property impacted by membrane curvature is the lateral diffusion of membrane components, which has a direct influence on the interpretation of experimental results obtained from techniques such as Fluorescence Correlation Spectroscopy (FCS) and Single Particle Tracking (SPT) that often assume planar geometries. ${ }^{14-16}$ Notably, the effects of the underlying curvature can be falsely interpreted by slowed-down or even anomalous diffusion, ${ }^{16-18}$ calling for further clarification by alternative approaches such as computer simulations.

Biological membranes undergo thermal fluctuations affecting their curvature. These fluctuations are generally considered to slow diffusion down by increasing the thickness or extending the geometric path. ${ }^{19}$ Taking them into account would require the treatment of changing surfaces. Instead, here, we restrain ourselves to work in the static membrane limit, ${ }^{20}$ which is an excellent approximation for structures such as the cristae in the endoplasmic reticulum (ER) or auditory outer hair cells. ${ }^{21,22}$ Another aspect of the classification of membrane surfaces is based on their curvature. The curvature of a surface can be completely described by two scalar fields, the Gaussian curvature $K(\mathbf{r})$ and the mean curvature $H(\mathbf{r})$. Caveolae and budding vesicles with nonzero Gaussian curvature have radii of few 
dozen to a hundred nanometers. ${ }^{23}$ Tubular structures such as those in the mitochondrion are examples of developable surfaces, that only have non-vanishing mean curvature. ${ }^{24}$ The ER is also of great interest due to its complex membrane topology of folded membranes and highly curved regions. ${ }^{25,26}$

Several studies employing continuum methods have investigated the effect of both mean and Gaussian curvature on biological membranes, ${ }^{20,27-29}$ resulting in two major conclusions. First, at the continuum level, the surface diffusion of particles can only depend on Gaussian curvature $K$, and not on mean curvature $H$. This is related to the fact that developable surface - surfaces with vanishing $K$-are isometric to a plane. ${ }^{27}$ Obviously, this is only true for diffusion along the surface, whereas any curvature, $K$ or $H$, will affect the diffusion coefficients measured in experiments that assume a planar membrane. Secondly, on surface regions with $K>0$ (elliptic paraboloids) diffusion is slowed down, while on region with $K<0$ (hyperbolic paraboloids) it is sped up. ${ }^{27,28}$ In addition to these observations, the ratio of the real and projected long-time diffusion coefficients are shown to closely follow a so called area-scaling law ${ }^{20}$ under a broad range of conditions. Details about the area-scaling law can be found in the Supporting Information.

A major shortcoming of all these continuum methods is that they cannot account for the effects of lipid packing or the inclusion of proteins or other membrane heterogeneities. These effects carry the possibility of mean curvature influencing surface diffusion through heterogeneous membrane structures. ${ }^{29}$ While such effects are explicitly inherent in molecular simulations, their analyses presents another kind of drawback. Namely, the majority of the current analysis tools fall short of dealing with the changing membrane normal in curved membranes. ${ }^{30}$

Here, we present an algorithm and a software that implements it, coined CurD, for the calculation of Mean Square Displacement (MSD) in molecular simulations of curved membranes. We apply the method to coarse-grained Martini ${ }^{31,32}$ simulations of phospholipid bilayers forming a vesicular bud-like membrane protrusion ("Budded") and an undulating 
wave-like surface ("Wave"). Although slightly over-emphasized, the mean and Gaussian curvatures of our simulated systems are not far from those present in some biological systems. All relevant details of the simulation can be found in the Supporting Information. The simulated systems and their curvatures are shown in Fig. 1. The membrane in the "Wave" system has only mean curvature, $H$, hence it is isomorphic to a plane. This is not true for the lipid bilayer in the "Budded" system, as it also possesses Gaussian curvature, K. An important attribute of Gaussian curvature is its insensitivity to the orientation of the constituents of the bilayer. A bowl-shaped (saddle-shaped) region always has positive (negative) Gaussian curvature, irrespective of whether the bud bulges into the cytoplasmic or the extracellular space. The curvatures (bottom panels of Fig. 1), while properly capture the topology of the system, exhibit some irregularities due to the use of mesh surfaces (see the Supporting Information). Even though their relative sign depends on the chosen leaflet, a strong correlation between the magnitudes of $H$ and $K$ is clearly apparent.

The actual displacements of particles constrained to move on a surface are best described using geodesic distances. ${ }^{27,28}$ Geodesic distances can be efficiently computed on mesh surfaces using specialized methods, even without explicitly storing or constructing the corresponding shortest path. ${ }^{33}$ One such algorithm is the Vertex-oriented Triangle Propagation (VTP) of Qin et al. ${ }^{34}$ that simultaneously computes all distances from a source vertex to all other vertices on the mesh. Therefore, as the first step of our approach, we create a triangular mesh surface from each of the leaflets of the simulated membranes, using the center of mass of the lipid. Then, we use the position of the closest vertex as an approximation of the surface position of the lipid centers of mass. For sufficiently fine meshes, the error introduced by this discretization is negligible. To make full use of VTP algorithm and avoid multiple evaluation of the individual source vertices, the displacements must be grouped in a particular way. Triplets of integers (start vertex, end vertex, lagtime) representing the path of particles are written to a binary file and suitably sorted. By performing the grouping, all paths starting or ending at a given vertex form a contiguous block, irrespective of the 

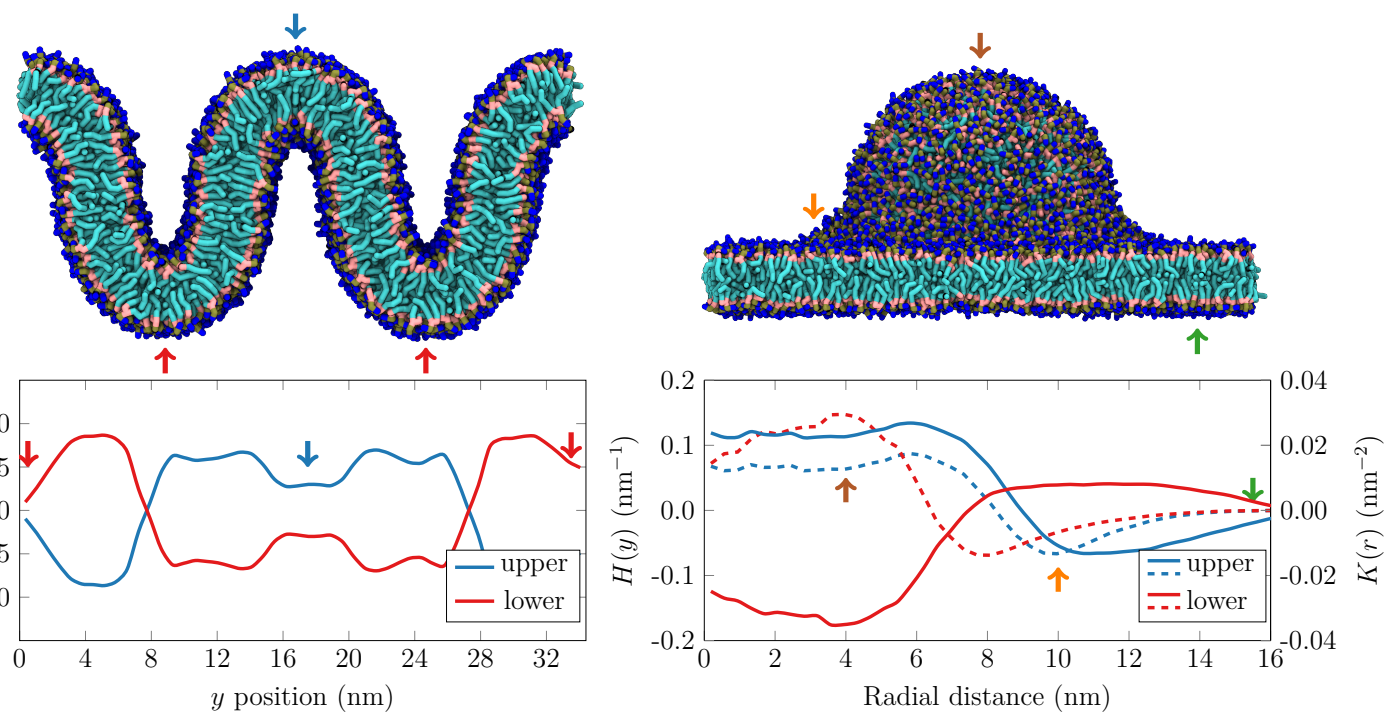

Figure 1: TOP: Snapshots of the simulated "Wave" (left) and "Budded" (right) system. BOTTOM: Average mean ( $H$, smooth lines) and Gaussian ( $K$, dashed lines) curvatures as a function of the position along the $y$ axis and the distance from the center of the bud, respectively. Blue line: upper leaflet, red line: lower leaflet. The membrane normal vector was always taken to point from the acyl chains towards the headgroups. The arrows indicate regions of given curvature. For a developable surface such as the "Wave" system, $H(y)=$ $\left(2 R_{y}(y)\right)^{-1}$ is the inverse of the diameter of the osculating cylinder to the surface at the corresponding value of $y$.

lagtime. Then, a single call of the VTP algorithm on the source vertex $i$ is sufficient to evaluate one complete block, as all the paths that start or end at vertex $i$ are grouped together in it, independently of the lagtime. Finally, the calculated distances are assigned both to the source and end vertices of the path, while keeping track of the lagtime. Once properly processed, the mesh contains the discretized spatial distributions of the geodesic MSD values at various lagtimes. Importantly, our approach is flexible enough to handle surface meshes of arbitrary shapes ${ }^{2,35}$ subject to the condition that they do not change in time. It is also modular, so that the distance calculation algorithm can readily be swapped with other existing methods. An illustration of the major steps of the algorithm is presented in Fig. 2. Details on the creation of the surface meshes, the magnitude of the discretization error, the handling of periodic boundary conditions and information about the scaling of the algorithm can be found in the Supporting Information. Here, we used meshes of 40,000 
points with a spacing of $\approx 0.4 \mathrm{~nm}$ to cover four periodic images, yet the algorithm scales reasonably well to meshes at least twice this size, corresponding to membranes with over 10,000 lipids. Independent calling of the VPT algorithm for different source vertices allows for further increase in studied system size trough the implemented trivial parallelization. For the sake of simplicity, the computed MSD curves are generally interpreted as $D_{\text {eff }}=\mathrm{MSD} / 4 \Delta$, even though this usually makes $D_{\text {eff }}$ time-dependent, and therefore somewhat ill-defined. ${ }^{27}$ Furthermore, for appreciable curvatures the time-dependence of $D_{\text {eff }}$ can be misinterpreted as anomalous diffusion. ${ }^{16}$

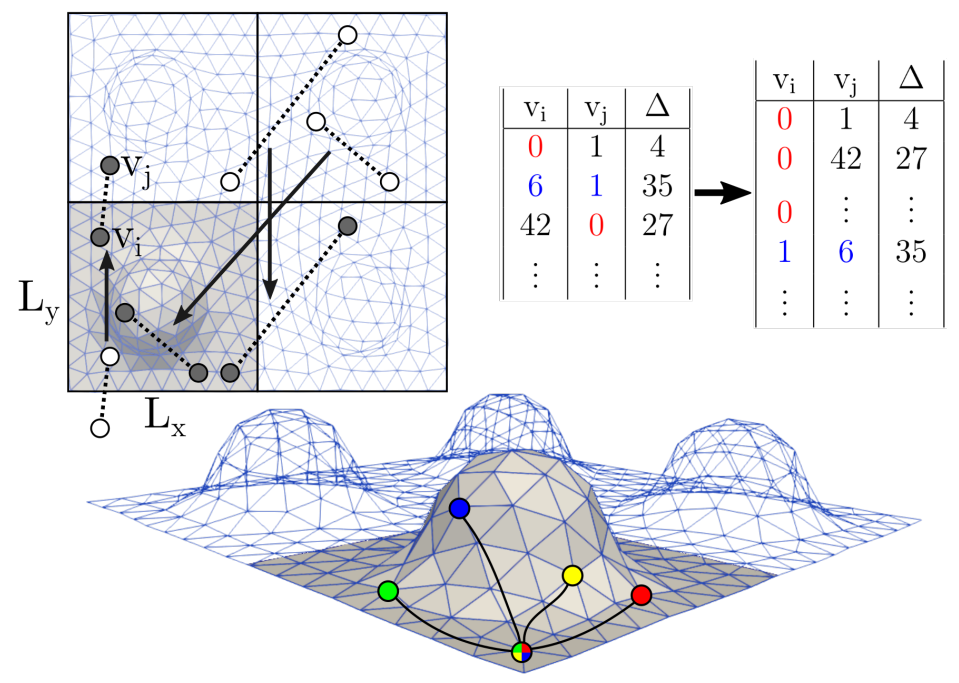

Figure 2: Illustration of the main steps of calculating geodesic Mean Square Displacements. After preparing a triangular mesh surface, all particle displacements, that is, initial and final particle positions (white circles) separated by lagtime are mapped onto the surface ensuring that both the starting and end point are on the mesh, as close to the origin as possible (gray circles). Particle displacements at large lagtimes require taking into account more periodic images. The resulting $v_{i}$ and $v_{j}$ pairs of mesh vertices are written to a binary file along with the corresponding lagtime, $\Delta$. To enable the efficient use of the VTP algorithm, all pairs are ordered so that the lower vertex index appears first (see for example the numbers highlighted in blue), and subsequently ordered along the first column. This way, all particle displacements involving a given $v_{i}$ appear as a single contiguous block. Finally, the VTP algorithm is needed to be called on each source vertex $v_{i}$ only once to evaluate all distances in the block. These evaluations are independent, therefore are readily parallelized, as done in the current implementation. The computed displacements are assigned to both vertices while also taking account of the associated lagtime. 
To investigate the differences between the various diffusion measurement methods, we evaluated the Mean Square Displacements both by projecting the motion of the particles onto the macroscopic plane of the membrane - as typical in numerous experimental approachesand by using our approach presented above. The projected and surface MSD values as a function of the position along the $y$ axis or the radial distance in case of the "Wave" and "Budded" systems are shown at a few selected lagtimes in Fig. 3 and 4, respectively.

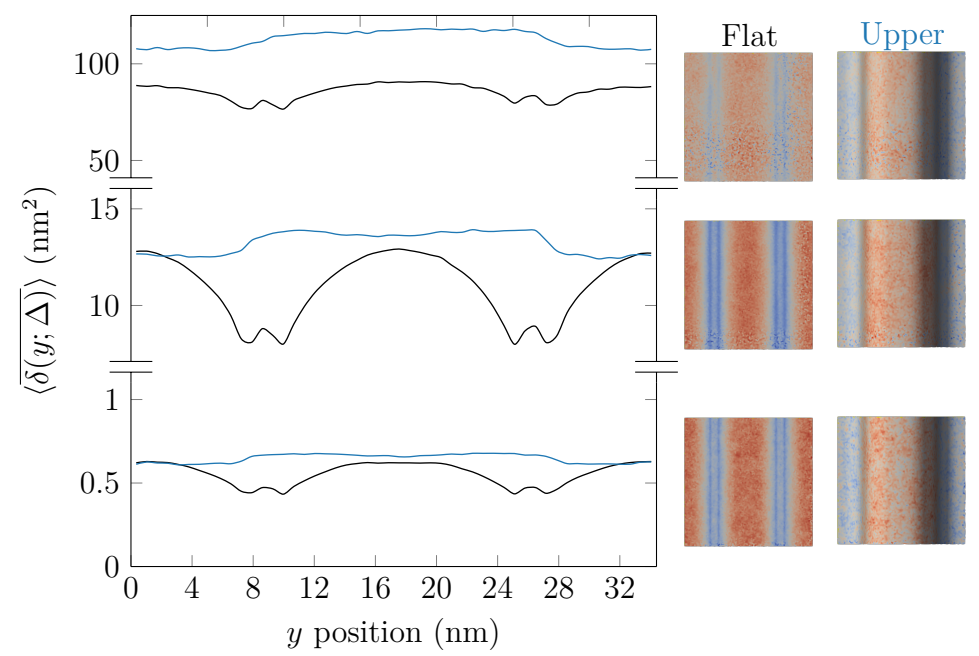

Figure 3: Two dimensional ("Flat", discarded z coordinate) and geodesic ("Upper" (for upper leaflet)) Mean Square Displacement values at selected lagtimes $(\Delta=2,53,453 \mathrm{~ns}$ from bottom upward) as a function of the position along the $y$ axis in the "Wave" system. The values in the "Flat" system are averaged across both leaflets. The "Lower" leaflet is a shifted version of the "Upper" leaflet, and as such, it is omitted. The images on the right illustrate the distributions of MSD values at the corresponding lagtimes.

In both systems, using the projected displacements has a major effect on the apparent motion of the molecules due to ignoring their motion along the axis perpendicular to the plane of the membrane. The magnitude of this effect is directly related to local orientation of the membrane segments, thus the projected displacements in the almost vertical regions of the "Wave" and "Budded" membranes underestimate the actual displacements the most. This phenomenon is purely geometric, and can have a significant impact on results obtained from experiments. The 2D MSD profiles (black lines) get progressively smoother with increasing lagtime due to the mixing of molecules originating from regions of different curvatures. 


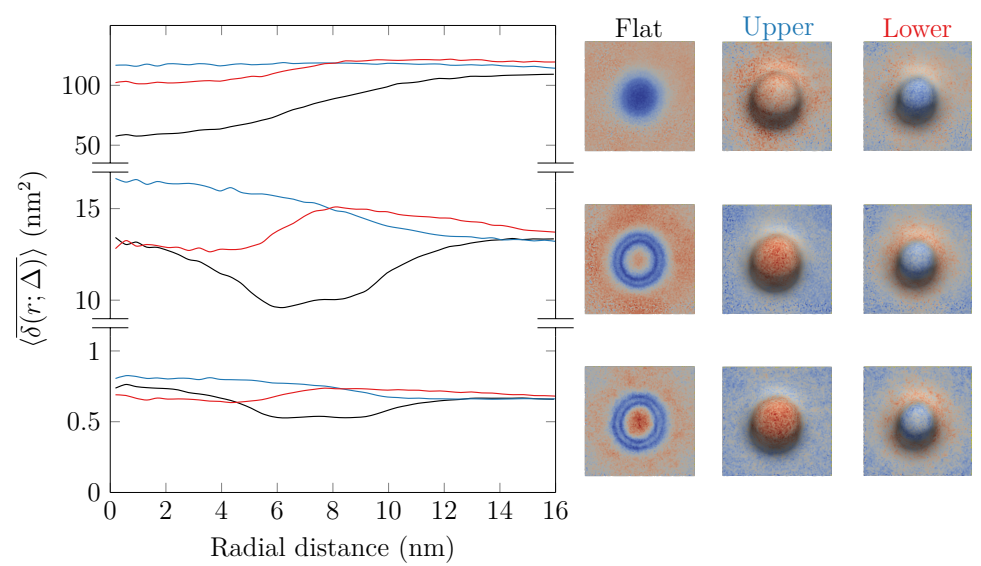

Figure 4: Two dimensional ("Flat", discarded z coordinate) and geodesic ("Upper" and "Lower") Mean Square Displacement values at selected lagtimes $(\Delta=2,53,453$ ns from bottom upward) as a function of the distance from the center of the bud in the "Budded" system. The values in the "Flat" system are averaged across both leaflets. The images on the right illustrate the distributions of MSD values at the corresponding lagtimes.

Consequently, on infinite periodically repeating lattices such as the membranes simulated in the current study, the influence of curvature on the projected diffusion coefficients can be approximated in the long lagtime limit as a simple geometrical scaling of the corresponding planar value, ${ }^{20}$ as discussed in the Supporting Information. In the "Budded" system, while at small lagtimes the center of the bud $(r=0)$ exhibits the largest displacements, this is completely obscured by the mixing with molecules originating from regions of lower apparent diffusion coefficient (the sides of the bud). Such a qualitative change in the apparent mobility of various regions has direct implications on the interpretation of experimental results on curved membranes, as it renders diffusion coefficients strongly time-dependent.

When comparing the projected and the geodesic displacements, it becomes clear that using the projected values manifests as an artificial slowdown on surfaces of both mean and Gaussian curvature, with a magnitude roughly proportional to the curvature. When the correct geodesic displacements are used, all MSD curves shift to higher values and become smoother as a function of the relevant coordinate. In addition, similarly to the conventional (projected) displacements, molecules originating from regions of different curvature 
gradually mix as the lagtime increases, producing uniform MSD profiles at large enough lagtimes. To better quantify these effects, we computed the diffusion coefficient distributions using the conventional 2D calculation method and the geodesic-based approach presented here. Furthermore, we also decomposed the latter based on local curvature (see Supporting Information for details). The diffusion coefficients at the individual mesh points were calculated at the lag time of $53 \mathrm{~ns}$, instead of separately applying linear regression to them. It must be noted, that computing a diffusion coefficient distribution on surface mesh points is not strictly equivalent to the per-particle distribution. However, the almost uniform density of lipid centers of mass (see Fig. S?? and S??) indicates that the distinction should be only minor. Indeed, the simple division using one lagtime for mesh point MSDs provided a very similar value to linear fits to MSD data calculated for lipids, validating our approach. The distributions of the diffusion coefficients in different membrane environments, shown in Fig. 5, confirm that assuming 2D movement significantly underestimates the diffusion coefficient of molecules moving on curved surfaces (compare Projected and Real). Additionally, while the Flat region of the "Budded" system corresponds to the "apparently fastest" domain in the Projected distribution (both are around $6 \cdot 10^{-7} \mathrm{~cm}^{2} / \mathrm{s}$ ), the Flat part of the "Wave" is conclusively faster than the projection. This is in agreement with the orientation of the planar membrane regions in the two systems (cf. Fig. 1).

Contrary to continuum theories predicting the lack of influence of mean curvature $H$ on surface diffusion, ${ }^{27-29}$ the "Wave" system seems to exhibit clear correlations between the motion of particles and the mean curvature in regions with $H>0$ and $H<0$ based on Fig. 3 and S??. However, based on Fig. 5, there is essentially no difference between the average diffusion coefficients on the Flat and Curved parts in the "Wave" system, thus leading to the apparent conclusion of $H$ not affecting lateral diffusion. Nevertheless, by further decomposing the mean curvature into positively and negatively curved regions, we can conclude that $H>0$ indeed enhances, while $H<0$ hinders diffusion. These two effects are opposite and equal in magnitude, giving rise to zero net change compared to the flat 
domains.

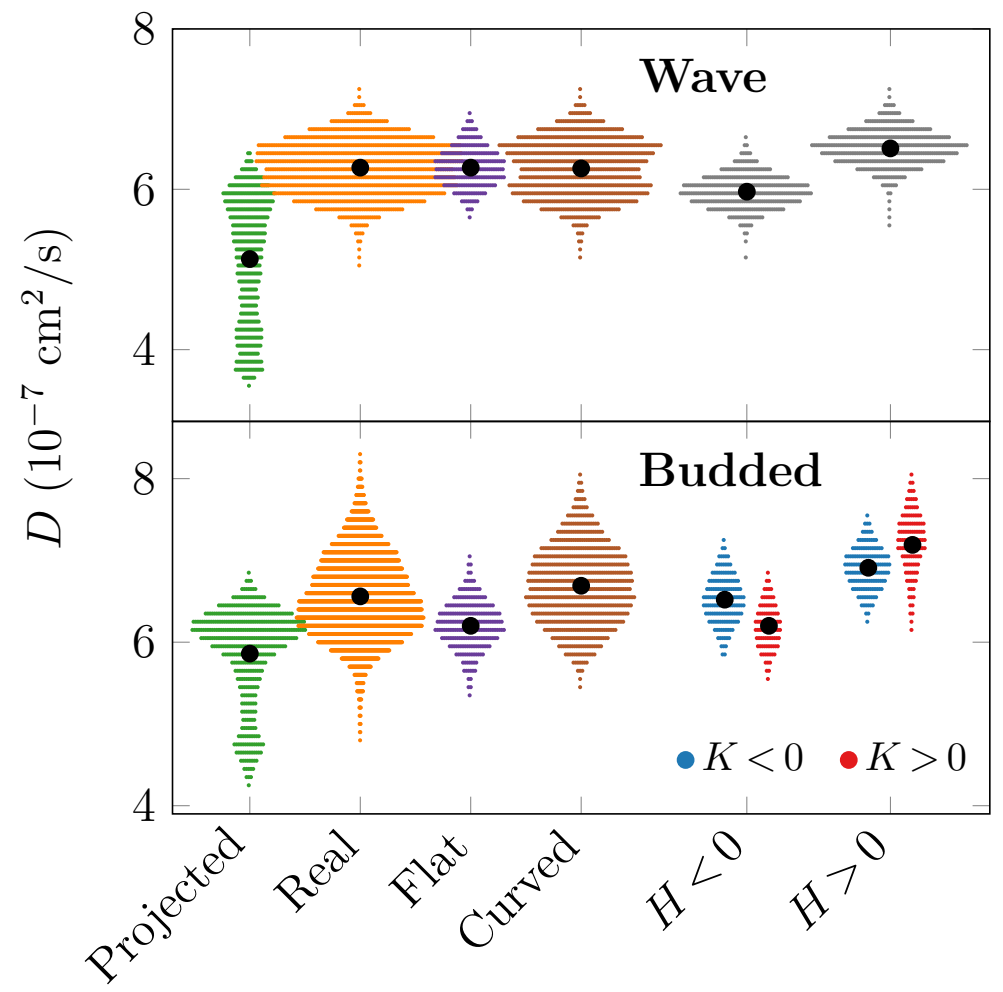

Figure 5: Spatial distributions of diffusion coefficients as determined by MSD $/ 4 \Delta, \Delta=53 \mathrm{~ns}$. TOP: "Wave" system, BOTTOM: "Budded" system. The Projected and Real coefficients were determined by the conventional 2D method and using the geodesic distances, respectively. The latter values were further subdivided into categories based on their curvature (See Supporting Information). $H$ : mean curvature, $K$ : Gaussian curvature. The black dots show mean values, and the area covered by the distributions is proportional to the prevalence of the corresponding curvature in the simulated system.

The particles in regions of higher mean curvature are generally less densely packed (see the Supporting Information) in agreement with the results of Yesylevskyy et al., ${ }^{13}$ and hence more mobile. The converse is true for particles in regions of negative mean curvature where the headgroups are more compressed. The observed discrepancy between continuum predictions and molecular simulations is a direct consequence of lipid packing effects, which are usually not included in the continuum models. ${ }^{29}$ Even though the differences are minor in the systems studied here, they can be more significant with e.g. a more complex lipid mixture where the different lipid species are sorted by curvature. 
Because there is a fundamental asymmetry in the curvature of its leaflets, in the analysis of the "Budded" system the individual leaflets must be treated separately. In case of the upper leaflet (blue curves in Fig. 4), positive Gaussian curvature, $K>0$, seems to correlate with faster diffusion, while regions with $K<0$ exhibit slower diffusion. This behavior is verified by the analysis demonstrated in Fig. 5, and again goes against the conclusion drawn from continuum theories, where $K>0$ results in slower diffusion and $K<0$ causes faster diffusion. ${ }^{28}$ Similarly to the mean curvature, the speedup in the upper layer can be ascribed to a less dense packing of lipids, at least at the level of headgroups.

Importantly, Gaussian curvature is insensitive to the direction of the membrane normal: bowls have positive and saddles have negative Gaussian curvature. Therefore, if the diffusion only depended on the Gaussian curvature, one would expect similar tendencies in both upper and lower leaflets. This is clearly not the case, as the lower leaflet seems to follow the theoretical prediction presented above. ${ }^{?}$ Consequently, the theoretically predicted role of Gaussian curvature is not the only factor determining surface diffusion in molecular systems, mean curvature also must play an important role. What is more, in our "Budded" system the absolute magnitude of Gaussian and mean curvatures positively correlate, while the mean curvature also encodes the orientation of the lipids. Thus, the mean curvature seems sufficient to explain the behavior observed in our simulations. Joined with the headgroup densities, the arising picture nicely follows that of the "Wave" system possessing only mean curvature. This line of reasoning is further supported by Fig.?? containing the correlations of the variables of interest.

To conclude, we have developed an algorithm and implemented it to analyze the diffusion dynamics along curved membrane surfaces. We have applied our method to two simulated membranes with different topologies - one with only mean curvature and another with additional Gaussian curvature. Our approach is able to resolve the roles of curvature and lipid packing on dynamics in multi-microsecond trajectories on systems spanning dozens of nanometers in size, i.e. in biologically relevant scales. This is a task only made possible 
with the efficient implementation of the distance calculation in our algorithm. The richness of molecular motion revealed by our analysis method show details of the lateral diffusion that are otherwise obscured by the use of conventional, projected diffusion coefficients. The present results not only shed light on the importance of lipid packing effects on the motion of the particles, but also indicate the fundamental way by which the mean curvature $H$ can affect diffusion. Based on the picture emerging following the analysis, either the mean curvature or the lateral density of lipid headgroups seems to be a reliable indicator of changes in lateral diffusion. Of course, the failure of the continuum models predicting the significance of Gaussian curvature $G$ on the length-scales of our simulated membranes is not unexpected. Due to the high degrees of curvature, the size of the lipid themselves becomes commensurate with the radius of curvature, increasing the relevance of lipid packing-related effects. The fundamentally different behavior of the upper and lower leaflets with respect to the Gaussian curvature has a significant conceptual impact on continuum theories ${ }^{27,28}$ and surface MC simulations. ${ }^{2,36,37}$

\section{Acknowledgement}

B.F. and M.J. acknowledge the support from the Czech Science Foundation (EXPRO Grant 19-26854X). M.J. thanks CSC-IT Center for Science for computational resources and the Academy of Finland (Postdoctoral researcher grant no. 338160) and Emil Aaltonen foundation for funding.

\section{Supporting Information Available}

All simulation inputs and outputs are freely available at the Zenodo repository under the DOI-s listed in the SI. Details on the analyses are provided in the SI and the implementation of the method is available at https://github.com/balazsfabian/curved-diffusion. 


\section{References}

(1) Singharoy, A.; Maffeo, C.; Delgado-Magnero, K. H.; Swainsbury, D. J.; Sener, M.; Kleinekathöfer, U.; Vant, J. W.; Nguyen, J.; Hitchcock, A.; Isralewitz, B. et al. Atoms to phenotypes: molecular design principles of cellular energy metabolism. Cell 2019, 179, 1098-1111.

(2) Pezeshkian, W.; König, M.; Wassenaar, T. A.; Marrink, S. J. Backmapping triangulated surfaces to coarse-grained membrane models. Nature communications 2020, 11, 1-9.

(3) Enkavi, G.; Javanainen, M.; Kulig, W.; Róg, T.; Vattulainen, I. Multiscale simulations of biological membranes: the challenge to understand biological phenomena in a living substance. Chemical reviews 2019, 119, 5607-5774.

(4) McMahon, H. T.; Boucrot, E. Membrane curvature at a glance. Journal of cell science 2015, 128, 1065-1070.

(5) Anderson, R. G. The caveolae membrane system. Annual review of biochemistry $\mathbf{1 9 9 8 ,}$ 67, 199-225.

(6) Colina-Tenorio, L.; Horten, P.; Pfanner, N.; Rampelt, H. Shaping the mitochondrial inner membrane in health and disease. Journal of Internal Medicine 2020, 287, 645664.

(7) Olzmann, J. A.; Carvalho, P. Dynamics and functions of lipid droplets. Nature reviews Molecular cell biology 2019, 20, 137-155.

(8) Dasgupta, R.; Miettinen, M. S.; Fricke, N.; Lipowsky, R.; Dimova, R. The glycolipid GM1 reshapes asymmetric biomembranes and giant vesicles by curvature generation. Proceedings of the National Academy of Sciences 2018, 115, 5756-5761.

(9) Mim, C.; Unger, V. M. Membrane curvature and its generation by BAR proteins. Trends in biochemical sciences 2012, 37, 526-533. 
(10) Woodward, X.; Kelly, C. V. Nanoscale membrane curvature sorts lipid phases and alters lipid diffusion. bioRxiv 2020,

(11) Baoukina, S.; Ingólfsson, H. I.; Marrink, S. J.; Tieleman, D. P. Curvature-Induced Sorting of Lipids in Plasma Membrane Tethers. Advanced Theory and Simulations 2018, 1,1800034 .

(12) Boyd, K. J.; May, E. R. BUMPy: a model-independent tool for constructing lipid bilayers of varying curvature and composition. Journal of chemical theory and computation 2018, 14, 6642-6652.

(13) Yesylevskyy, S. O.; Rivel, T.; Ramseyer, C. The influence of curvature on the properties of the plasma membrane. Insights from atomistic molecular dynamics simulations. Scientific reports 2017, $7,1-13$.

(14) Kabbani, A. M.; Woodward, X.; Kelly, C. V. Revealing the effects of nanoscale membrane curvature on lipid mobility. Membranes 2017, 7, 60.

(15) Adler, J.; Sintorn, I.-M.; Strand, R.; Parmryd, I. Conventional analysis of movement on non-flat surfaces like the plasma membrane makes Brownian motion appear anomalous. Communications Biology 2019, 2, 12.

(16) Gesper, A.; Wennmalm, S.; Hagemann, P.; Eriksson, S.-G.; Happel, P.; Parmryd, I. Variations in Plasma Membrane Topography Can Explain Heterogenous Diffusion Coefficients Obtained by Fluorescence Correlation Spectroscopy. Frontiers in Cell and Developmental Biology 2020, 8, 767.

(17) Metzler, R.; Jeon, J.-H.; Cherstvy, A. G.; Barkai, E. Anomalous diffusion models and their properties: non-stationarity, non-ergodicity, and ageing at the centenary of single particle tracking. Physical Chemistry Chemical Physics 2014, 16, 24128-24164. 
(18) Adler, J.; Shevchuk, A. I.; Novak, P.; Korchev, Y. E.; Parmryd, I. Plasma membrane topography and interpretation of single-particle tracks. Nature Methods 2010, 7, 170171.

(19) Reister, E.; Seifert, U. Lateral diffusion of a protein on a fluctuating membrane. EPL (Europhysics Letters) 2005, 71, 859.

(20) Naji, A.; Brown, F. L. Diffusion on ruffled membrane surfaces. The Journal of chemical physics 2007, 126, 06B611.

(21) Boal, D.; Boal, D. H. Mechanics of the Cell; Cambridge University Press, 2012.

(22) Slepecky, N.; Ulfendahl, M.; Flock, Å. Effects of caffeine and tetracaine on outer hair cell shortening suggest intracellular calcium involvement. Hearing research 1988, 32, $11-21$.

(23) Meyer, H. W.; Westermann, M.; Stumpf, M.; Richter, W.; Ulrich, A. S.; Hoischen, C. Minimal radius of curvature of lipid bilayers in the gel phase state corresponds to the dimension of biomembrane structures "caveolae". Journal of structural biology 1998, $124,77-87$.

(24) Ölveczky, B. P.; Verkman, A. Monte Carlo analysis of obstructed diffusion in three dimensions: application to molecular diffusion in organelles. Biophysical journal 1998, $74,2722-2730$.

(25) Almeida, P. F.; Vaz, W. L. Handbook of biological physics; Elsevier, 1995; Vol. 1; pp 305-357.

(26) Sbalzarini, I. F.; Hayer, A.; Helenius, A.; Koumoutsakos, P. Simulations of (an) isotropic diffusion on curved biological surfaces. Biophysical journal 2006, 90, 878-885.

(27) Faraudo, J. Diffusion equation on curved surfaces. I. Theory and application to biological membranes. The Journal of chemical physics 2002, 116, 5831-5841. 
(28) Yoshigaki, T. Theoretically predicted effects of Gaussian curvature on lateral diffusion of membrane molecules. Physical Review E 2007, 75, 041901.

(29) Gov, N. S. Diffusion in curved fluid membranes. Physical Review E 2006, 73, 041918.

(30) Yesylevskyy, S.; Ramseyer, C. Determination of mean and Gaussian curvatures of highly curved asymmetric lipid bilayers: the case study of the influence of cholesterol on the membrane shape. Physical Chemistry Chemical Physics 2014, 16, 17052-17061.

(31) Marrink, S. J.; De Vries, A. H.; Mark, A. E. Coarse grained model for semiquantitative lipid simulations. The Journal of Physical Chemistry B 2004, 108, 750-760.

(32) Marrink, S. J.; Risselada, H. J.; Yefimov, S.; Tieleman, D. P.; De Vries, A. H. The MARTINI force field: coarse grained model for biomolecular simulations. The journal of physical chemistry B 2007, 111, 7812-7824.

(33) Crane, K.; Livesu, M.; Puppo, E.; Qin, Y. A Survey of Algorithms for Geodesic Paths and Distances. arXiv preprint arXiv:200\%.10430 2020,

(34) Qin, Y.; Han, X.; Yu, H.; Yu, Y.; Zhang, J. Fast and exact discrete geodesic computation based on triangle-oriented wavefront propagation. ACM Transactions on Graphics (TOG) 2016, 35, 1-13.

(35) Bhatia, H.; Ingólfsson, H. I.; Carpenter, T. S.; Lightstone, F. C.; Bremer, P.-T. MemSurfer: a tool for robust computation and characterization of curved membranes. Journal of chemical theory and computation 2019, 15, 6411-6421.

(36) Christensen, M. How to simulate anisotropic diffusion processes on curved surfaces. Journal of Computational Physics 2004, 201, 421-438.

(37) Pezeshkian, W.; Marrink, S. J. Simulating realistic membrane shapes. Current Opinion in Cell Biology 2021, 71, 103-111. 